\title{
An Overview of Intrusion Detection and Prevention Systems (IDPS) and Security Issues
}

\author{
Ahmad Sharifi ${ }^{1}$, Freshteh Farokh Zad ${ }^{2}$, Farnoosh Farokhmanesh ${ }^{3}$, Akram \\ Noorollahi ${ }^{4}$, Jallaledin Sharifi ${ }^{5}$ \\ ${ }^{1}$ School Of Information Technology, Jawaharlal Nehru Technological University, India \\ ${ }^{2}$ Department Of Information Technology, Guahati University, India \\ ${ }^{3}$ Department Of Information Technology and Services, Universitat Politècnica de Catalunya, Spain \\ ${ }^{4}$ Department Of Management, Payam-e-Noor University, Aliabad, Iran \\ ${ }^{5}$ IT Section, Sharif Network Designers Company, Shahroud, Iran
}

\begin{abstract}
Technical solutions, introduced by policies and implantations are essential requirements of an information security program. Advanced technologies such as intrusion detection and prevention system (IDPS) and analysis tools have become prominent in the network environment while they involve with organizations to enhance the security of their information assets. Scanning and analyzing tools to pinpoint vulnerabilities, holes in security components, unsecured aspects of the network and deploying of IDPS technology are highlighted.
\end{abstract}

Keywords: Detection, intrusion, prevention, security, vulnerability

\section{INTRODUCTION}

With computer systems increasingly under attack, information security is more serious in user views "Fig.1". Security protects computer and everything associated with it including networks, terminals, printers, cabling, disks and most important, it protects the available information in this environment. Today, users are the content [1], [2]. Driving the growth, and at the same time being driven by it, the explosion in computer networks is expanding the impact of the social web. The way that content is shared and accessed, is now the core of a new global culture, affecting and combining the spheres of personal and business life. The purpose is to protect against intruders who break into systems to catch sensitive data, steal passwords, or whatever misuses they can behave. Confidentiality, integrity and availability are three distinct aspects in the security domain. Protection of information is ensured by confidentiality. Avoiding from corruption the information and permission to unauthorized access or malicious activity is achieved by integrity. Availability assures efficiency of working and ability for recovery in disaster situations. The security of the overall system is restricted by the safety of its weakest link. Any single weakness can affect the security of the system entirely. The threats include the web site defacement, network penetrations cause corruption and loss of data, denial of service attacks, viruses, Trojans and endless series of new stories proves that the threats are real. In fact, network security involves with three realities: first, the defender has to defend against every possible attack, while the attacker only needs to distinguish one weakness. Second, the immense complexity of modern networks forms them impossible to secure properly. Third, professional attackers may encapsulate their attacks in programs, allowing ordinary people to use them. According to study vulnerability assessments and intrusion prevention or intrusion detection is just one aspect of IT security management. However, due to recent developments with the continuing spread of network connectivity IT security management, is faced with yet another challenge, requiring a structured approach for an adequate response. In the real world, security includes prevention, detection and response. Perfect prevention means no requirement for detection even though due to lack of pure prevention, this is not applicable, especially in computer networks. 


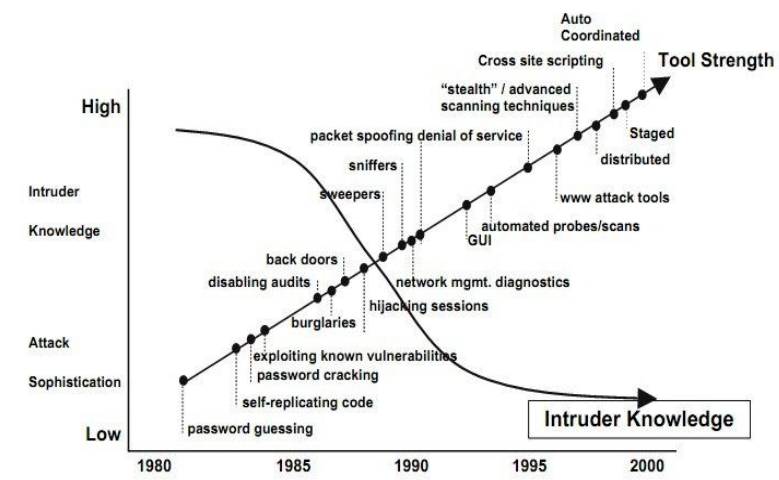

Fig 1. Attack sophistication versus intruder technical knowledge in past decades

\section{THREATS, VULNERABILITIES AND INTRUSIONS}

Whereas, digital information is represented by zeroes and ones, they can be threatened by unauthorized accessibility. So proportional methods like environmental, personal, and administrative security must be involved in this issue. The most obvious threat to an information resource is missing data, which almost caused by malicious behaviors internally or externally [3], [4]. Any authorized user who performs unauthorized actions that result in loss of control of computing assets, or actions resulting in unauthorized disclosure of information, is malicious insider one. In addition, these actions negatively impact the confidentiality, authenticity, and availability of information systems and information assets. The term vulnerability, describes a problem, i.e., a programming bug or common configuration error that allows a system or network to be attacked or broken into, mostly caused by complexity of networks. More precisely, vulnerability is a security hole, i.e., hardware, software or network weakness which allows an attacker to reduce an information assurance. Indeed, finding vulnerabilities is a main part of the hacker to attack. Security holes can be seen as open doors where they are thought to be closed. Poor passwords, bugs of software, a computer virus or a script code injection and weak links may result in vulnerabilities. According to the report, the number of vulnerabilities discovered in September 2011 is still on the rise with an increase of more than 70\% over the past two years. Intruder and virus are named as two more popular threats in security and commonly addressed to as a hacker or cracker. Intruders can be classified into three classes. First is masquerader, an individual who pretends themselves as legitimate accessibility to exploit the legitimate user account. Second is misfeasor, a legitimate user who access to unauthorized data, programs and resources or who is authorized for such access but misuse his or her privileges. Third is clandestine user, an individual who seizes supervisory control of the system and uses this control to evade auditing and access controls to surpass audit collection. The masquerader is likely to be an outsider; the misfeasor commonly is an insider, and the clandestine user can be either an outsider or an insider. Password guessing attack to capture passwords, modifying to log information, spoofing data and other new daily produced mechanisms using malware; spywares, Trojans will tend to hand the data to the attacker more reliable than previous methods familiarized by a security guard of environments. According to report of security companies, traditional email spam still remains a significant problem especially in business. Users still need to keep their inboxes clear of junk and advanced mail filtering systems are a requirement in any business hoping to use email efficiently. All kinds of tricks are visible in social engineering to lure its victims. A good first step to combat the unknown threat is user education, but even informed efforts occasionally are hindered by unwise activities from legitimate parties. These kinds of threats are serious challenge facing businesses.

\section{INTRUSION DETECTION AND PREVENTION SySTEM CONSIDERATIONS}

\section{Definition}

Attempts to breach information security are rising every day, along with the availability of the vulnerability assessment tools that are openly available on the Internet free, as well as for commercial use [5]. An intrusion occurs while an attacker attempts to get into an information system or cause disruption; in fact, its behavior intends to do harm. Intrusion detection and prevention systems help information system prepare for, and deal with attacks. They accomplish this by collecting information from a diversity of systems, monitoring and then analyzing for possible security problems. Indeed, an intrusion detection system (IDS) after detection of a violation raises an audible or visual alarm, or it can be silent like an e-mail message or pager alert. Another extension of this technology is the intrusion prevention system (IPS), which can detect an intrusion and in addition prevent that intrusion to be successful by an active response. 


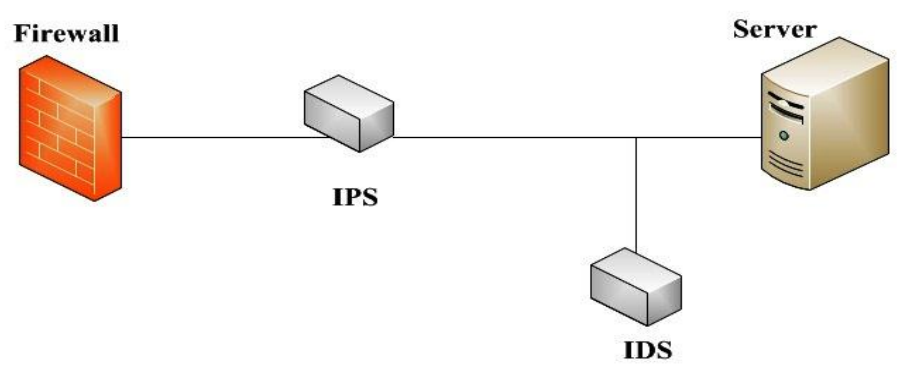

Fig 2. IDS and IPS systems application

Whereas the two systems often coexist "Fig.2", the combined term intrusion detection and prevention system (IDPS) is commonly used to describe current anti-intrusion technologies. As IDPS terminology point of view, some standard terms list as below:

Alert: Raising an alert in the form of audible signals, e-mail messages, page notifications or pop-up windows. Evasion: Changing format by an attacker to avoid from detecting by IDPS.

False negative: Failure of detecting a real attack by IDPS, while the main function of IDPS is detect and respond to attacks.

False attack stimulus: Triggering of alert by an event in the absence of an actual attack.

False positive: Raising alert by IDPS in the absence of an actual attack. They tend to pervasive users to be insensitive to alerts so, they reduce their activity to real intrusion events.

Noise: Alarm events that are accurate but do not pose significant threats to information security. Unsuccessful attacks are the most popular source of IDPS noise.

Site policy: Configuration and policy prepared by organization for implementation of IDPS.

Site policy awareness: So-called smart IDPS means the ability of IDPS to dynamically modify its configuration in response to environment activity.

True attack stimulus: Alarm triggering by an event and causes an IDPS to react as if a real attack is in progress.

Tuning: The process of adjusting an IDPS to maximize its efficiency in order to detect true positives while minimizing both false positive and false negatives.

Confidence value: Measurement of correct detections by IDPS and identify certain types of attacks.

Alarm filtering: Classification of IDPS alerts so that they are manageable more efficient. They are similar to packet filters and they can filter items by source and destination through operating systems, confidence values and alert types.

Alarm clustering and compaction: Categorization of similar behavioral alerts on time into a single higherlevel alarm. This clustering may be based on a combination of frequency, attack signature or target similarity leads to reducing the number of alerts generated.

Preventing from bad behaviors caused by misused information and detection of attacks and security violations not prevent by other measures is main duty of IDPS. Furthermore, [6] it involves with documentation of existing threats and being a controller for security design. By providing information about intrusions, advanced diagnosis, recovery, and various investigations allowable to perform for ongoing events including stopping the attack, terminating the network connection or user session, blocking the target accessibility, changing security.

\section{Types of IDPS and operations}

Two kinds of operations, including network-based and host-based systems are famous working domains which numerous of ongoing interesting patterns, are monitored by them. Based on functionality, anomaly based and signature based, sign to address [7], [8], [9]. Anomaly based one require making a decision, if the behavior of the system is the statistically significant departure from normal like; validity, the ratio of a TCP connection, payload, number of accesses to specific files and login frequency. Signature based one works through recognizing specific patterns of events or behavior that portend or go along with an attack. A host based IDPS (HIDPS) is typically implemented in software and resides on top of the operating system. On the other hand, they rely on the events collected by the hosts they monitor. The duty is monitoring of the internal behavior of the host such as the sequence of system calls made, file accessed and other methods using system or application logs and operating system audit trails to identify relevant events to an intrusion. Network based intrusion detection prevention systems (NIDPSs) gather input data by monitoring network traffic i.e., packets captured by network interfaces in promiscuous mode "Fig.3". While a situation occurs that the NIDPS designing for recognizing as an attack, it responds by sending notifications to the administrators. 


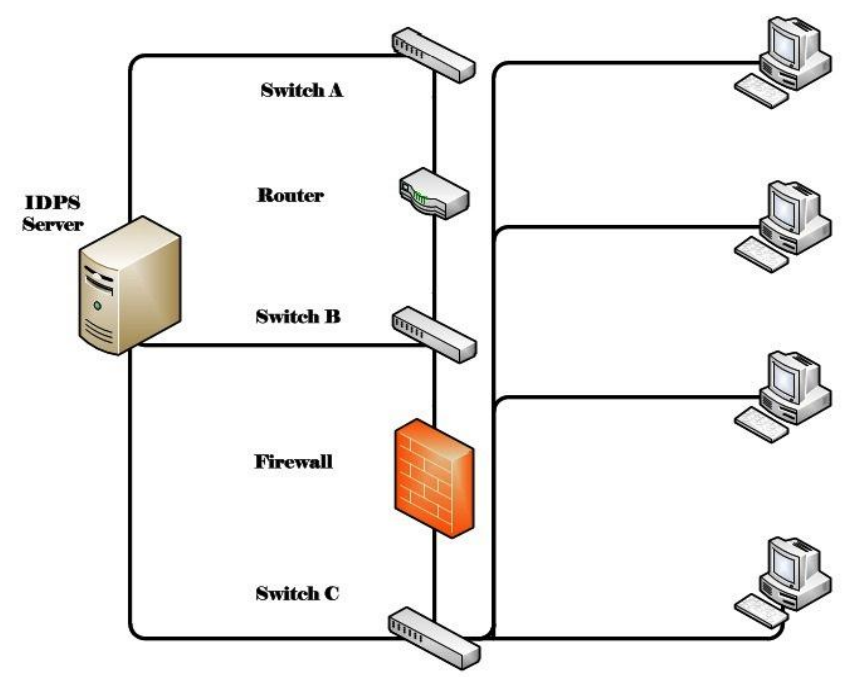

Fig 3. IDPS deployment in a network environment [10], [11].

NIDPSs are installed at a specific place in the network, i.e., on the inside of an edge router from where it is possible to look for the traffic going into and out of a particular network part. They deploy to inspect a special category of host computers on a particular network part, or its installation for monitoring the network traffic entirely. In a wireless environment, IDPS monitors and analyzes wireless network traffic, finding potential problems with the wireless protocols. Furthermore, wireless access point may contain IDPS capability in order to monitor. Wireless local area networks (WLANs) have exposed many different security flaws, aligning from misconfiguration of wireless access points (WAPs) to unreliable cryptographic algorithms. Mobile ad hoc networks are notably vulnerable because of their openness, varying topology, and lack of centralized management. The major important difference indicates today's NIDPSs rely on real-time traffic analyses upon a fixed infrastructure and so cannot function well in the mobile computing environment. NIDPSs are inclined to a salient ratio of false positive alerts. They are able to catch many but not all intrusions and alternatively, they are intrinsic to the nature of detecting intrusions in a network. In addition, their effectiveness depends limitedly on the specific design of the network infrastructure. Automatic response mechanisms, can be circumvented and exploited against the NIDPS owner to provoke denial of service conditions. Numerous methods exist that are able to evade detection. Hence, these systems must not consider in an isolated manner. NIDPSs are a valuable cover of security protection within a defense-in-depth planning, however their flaws will have to be mitigated by further security layers and technologies. Whereas, many attacks occur so quickly, automated mechanisms designed to thwart them may not be able to stop from initially succeeding. In these situations, intrusion prevention mechanisms often attempt to prevent the attack from spreading any further. Speed and accuracy are desirable features for an IDPS. High sensitivity implies a low false negative rate, whereas high selectivity implies a low false positive rate. The strategy for deploying an IDPS must be taken into account of factors, knowing how the IDPS will be managed and where position it need to be placed. The factors include number of administrators needed to install, configure and monitor as well as the number of management workstations, the size of the storage needed for retention of the data generated by the systems, and the ability of the organization to detect and respond to remote threats. IDPS control strategies include centralized control strategy, fully distributed control strategy and partially distributed control strategy are different methods to deploy. In all circumstances, designers have to select a deployment strategy based on a careful analysis of IT infrastructure requirements. Test facility has a significant capacity in this issue whereas, training scenarios can be developed collaborating monitoring of operations allowing users to recognize and respond to common attack circumstances. Capabilities and their specifications list in Table 1 and Table 2.

Table 2. Information gathering capabilities

\begin{tabular}{llc}
\hline Capability & Method & Result \\
\hline Identifying hosts & $\begin{array}{l}\text { Ability to create a list of hosts by IP } \\
\text { address or MAC address. }\end{array}$ & Hosts profile \\
& $\begin{array}{l}\text { Recognizing of analyzing techniques } \\
\text { Identifying Operating }\end{array}$ & $\begin{array}{c}\text { OS versions to identify } \\
\text { host vulnerable }\end{array}$ \\
$\begin{array}{l}\text { System } \\
\text { Identifying Application }\end{array}$ & $\begin{array}{l}\text { Keeping track of ports and } \\
\text { monitoring }\end{array}$ & $\begin{array}{c}\text { Identify potential } \\
\text { vulnerable application }\end{array}$ \\
$\begin{array}{l}\text { Identifying network } \\
\text { characteristic }\end{array}$ & $\begin{array}{l}\text { Collect traffic information like } \\
\text { number of hops in a path }\end{array}$ & $\begin{array}{l}\text { Awareness changing } \\
\text { network configuration }\end{array}$ \\
\hline
\end{tabular}


NIDPS products provide a broad diversity of security capacities. They normally carry out extensive logging of data connected to detect events. This data confirm the validity of alerts, to explore incidents, and to correlate events between the IDPS and other logging origins. Perfectly, network based IDPSs would be able to define all network actions like the runs of endpoints. Stateful protocol diagnosis techniques often attempt to prepare this by replicating the processing executed by collective types of clients and servers. This allows the IDPS to improve their detection accuracy slightly.

Table 3. Logging capabilities

\begin{tabular}{ll}
\hline Capability & Application \\
\hline Timestamp & Date and time \\
Connection ID & $\begin{array}{l}\text { Unique number assigns to TCP or other } \\
\text { session }\end{array}$ \\
Event or alarm type & $\begin{array}{l}\text { Realizing of occurrence types } \\
\text { Rating }\end{array}$ \\
Priority, severity, impact, confidence \\
Protocols & $\begin{array}{l}\text { Network, transport, and application layer } \\
\text { Source and destination IP address, TCP, }\end{array}$ \\
Path & UDP or ICMP \\
Number of bytes transmitted over a \\
Transmission & connection \\
Decoding payload & Application requests and responses \\
State information & Like authenticated user \\
Prevention & Avoiding action performed, in the \\
& required state \\
\hline
\end{tabular}

Many attackers utilize client and server-specific processing attributes like handling character encoding in their attacks as evasion techniques. While network-based IDPSs offer extensive detection capabilities, they have some significant restrictions including analyzing encrypted network traffic, handling high-traffic loads, and resisting attacks against the IDPSs themselves. Attacks through encrypted network traffic, virtual private network (VPN) connections, HTTP over SSL (HTTPS) and the SSH session typically cannot be detectable by NIDPS. The environment or interference in attack content are some response techniques. One critical issue is how to detect unknown patterns of attacks without generating too many false remains.

\section{Necessity of IDPS in organizations}

A simple principle of network security is a defense in-depth, which is a layered solution to secure valuable information on the network from malicious attacks using IDPS. After selection appropriated IDPS, it must be properly and efficiently deployed throughout the organization [12]. This job is performed through the design of IDPS strategy and issuing IDPS policy. Some factors like; the quantity of network traffic limitations, permit for configuration, installation requirements and frequencies of logs undergo for monitoring make document policy. The plan requires to create the policy will strengthen the communication between company management and security officials. Meanwhile, this will authorize organizational units to identify and solve conflicts before they become obstacles to successful IDPS deployment. Organizations should assimilate this policy into their comprehensive security policy or corporation rules and orders. Usage of incident response guidelines, staffing, setting, training and update signatures must be considered to imply as plan and economical resources for an effective deployment. In large companies more endpoint machines, servers and network segments indicate a more complicated setup and a longer installation time. Due to numerous computers and geographical diversity, planning plays a dominant role in a large enterprise IDPS deployment. The other well-known issue facing big companies includes scalability and the agent ratio. Considering the number of monitoring employees the IDPS managers for output, the employee skill and comfort level, the number of intrusion alerts per minute, the IDPS software carried out, and various other elements, the ideal agent ratio can alter from 5:1 to 50:1. Utilizing a subsection for testing aim or doing research at firms with a similar size, network structure, and intrusion risk may ease to pinpoint the optimal agent ratio when scaling for big companies.

\section{CONCLUSION}

Information security has become a legitimate concern for both organizations and computer users due to the growing confidence with computers and electronic transactions. Different techniques are used to support the security of an organization against threats or attacks. On the other side, attackers are discovering new techniques and ways to break these security policies. Firewalls, antivirus and antispyware are limited to provide security to the system against threats. The only way to strike them is realizing about their techniques to use for the attack. Therefore, security of organizations will have to adopt such a firmest model or mechanism, which provides strongest protection against threats to ensure that the system will remain secure. IDPS provides the facility to 
detect and prevent from attacks by inheriting multiple approaches. Active IDPS seeks to restrict the damage that attackers can penetrate by making the local network resistant to appropriate use.

\section{REFERENCES}

[1] U. A. Sandhu, S. Haider, S. Naseer and O. U. Ateeb, A Survey of Intrusion Detection \& Prevention Techniques, International Conference on Information Communication and Management IPCSIT: IACSIT Press, Singapore 2011.

[2] K. Scarfone and P. Mell, Guide to Intrusion Detection and Prevention Systems (IDPS), Recommendations of the National Institute of Standards and Technology: NIST Special Publication, 2007.

[3] B. Menezes, Network Security and Cryptography (Patparganj, New Delhi: Cengage Learning India Pvt. Ltd, 2010).

[4] J. R. Vacca, Computer and information security handbook (New York: Morgan Kaufmann, Elsevier 2009) 39-66, 133-166, 255267, 293-306, 349-393, 469-496.

[5] A. Fuchsberger, Intrusion Detection Systems and Intrusion Prevention Systems, Published by Elsevier: Information Security Technical Report 10, 2005, 134-139.

[6] P. Innella, http://www.symantec.com/connect/articles/managing-intrusion-detection-systems-large-organizationspart-one.

[7] EC-Council, Ethical Hacking and Countermeasures Version 6 Module XVII Web Application Vulnerabilities: International Council of E-commerce Consultants, 2008.

[8] R. E. Overill, ISMS insider intrusion prevention and detection, Published in Elsevier, Information security technical report 13, 2008, 216-219.

[9] N. Godbole, Information Systems Security: Security Management, Metrics, Frameworks and Best Practices (New Delhi: WIELY INDIA, 2009).

[10] N. F. Mir, Computer and Communication Networks (New York: Prentice Hall, 2006), 57-60, 101-125.

[11] B. Forouzan, Data Communications and Networking (New York: McGraw Hill, 2006), 3-23, 395-464.

[12] A. S. Ashoor and S. Gore, Importance of Intrusion Detection system (IDS), International Journal of Scientific and Engineering Research, 2(1), 2011, 1-4.

\section{Author's profile}

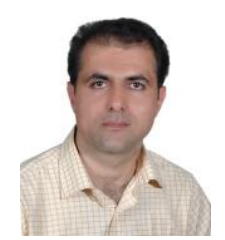

Ahmad Sharifi. He has received M. Tech in Computer Networks and Information Security from Jawaharlal Nehru Technological University (JNTU), Hyderabad, India. In addition, he has received his Bachelor in Electronic engineering from the industrial University of Shahroud, Iran. Ahmad has professional experiences on technical engineering on ISP and network designs for many years. In addition, he is involved with teaching in universities. He interests in Cryptography, WSN, ADHOC, MATLAB, OPNET and other related issues. His personal website is www.ahmadsharifi.com. Furthermore, he cooperates with RIPE NCC www.ripe.net.

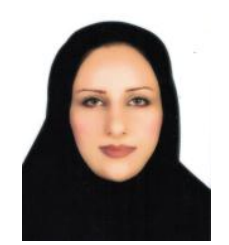

Fereshteh Farokh Zad. She received her B.E. degree from Azad university of Iran and M. Tech of IT from Jawaharlal Nehru Technological University (JNTU), Hyderabad, India. She is doing PhD at Guahati University in Assam, India. Her research interest includes distributed systems, networks and cloud computing.

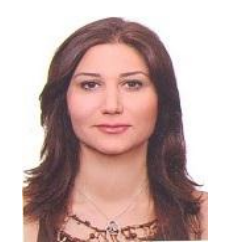

Farnoosh Farokhmanesh. She has received the Bachelor's degree from the Rajaee Teacher Training University, Iran. She worked as a technical ADSL support at Shatel ISP in 2009-2010, Iran. She is studding master in computer architecture in Universidad Politécnica de Cataluñ, Spain. She is a researcher on Wireless Sensor Network (WSN) at UPC University.

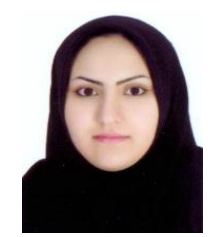

Akram Noorollahi. She has received the Bachelor's degree from Payam-e-Noor University of Aliabad, Golestan, Iran. She is an expert in Microsoft Project and IT management. In addition, she has worked with IT industry to manage projects, designing and marketing.

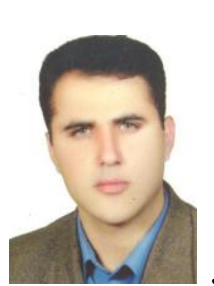

Jallaledin Sharifi. He is a Microsoft engineer. He has obtained MCITP 2008, besides of other experts and certificates in Cisco Technologies, MikroTik Routers and wireless, Linux based servers, WLAN, IPV4 and IPV6. In addition, he is chief of Sharif Network Designers Company http://www.sharifisdp.ir that includes Internet Service Providing and Distribution of services to consumers. Also he is a member of RIPE NCC http://www.ripe.net. Network topologies and infrastructure to the best quality of service in IT domain are considered as his professions. He designs for performance of modern services to enterprises. 